\title{
Two-dimensional numerical analysis of electroconvection in a dielectric liquid subjected to strong unipolar injection
}

\author{
Ph. Traoré ${ }^{1}$ and A. T. Pérez ${ }^{2}$ \\ ${ }^{1}$ Institut PPRIME, Boulevard Pierre et Marie Curie, BP 30179,86962 \\ Futuroscope-Chasseneuil, France \\ ${ }^{2}$ Departamento de Electrónica y Electromagnetismo, Facultad de Física, Avenida Reina \\ Mercedes s/n, 41012 Sevilla, Spain
}

(Received 4 April 2011; accepted 10 January 2012; published online 8 March 2012)

\begin{abstract}
Two-dimensional numerical simulations are carried out to examine the problem of transient electroconvection stability of dielectric liquids subjected to unipolar injection. The entire set of electrohydrodynamics equations associated with the electroconvective phenomena that occur in a layer of a dielectric liquid between two parallel electrodes subjected to a potential difference are solved numerically. We first validate the numerical simulation by comparing our linear stability electroconvection criteria with those obtained by other authors with a stability approach. In this paper, we restrict the study to the strong injection case, which corresponds to values of the non-dimensional injection parameter $C$ greater than or equal to 10 . The numerical solution of the electroconvective problem is then presented for rigid lateral boundary conditions. A detailed analysis of the scenario that occurs for different characteristic values of the stability parameter $T$ is provided. The flow structure and its behaviour highlight the existence of different regimes, from laminar to chaotic. The development of charged plumes has been observed in particular. We compute the electrical Nusselt number for different values of the stability parameter and ion mobility. The electrical Nusselt number saturates with increasing $T$, a fact that it is in agreement with available experimental data. Finally, a spectral analysis is conducted for different aspect ratios of the computational domain. The spectral analysis gives an insight into the physical origin of the velocity and current oscillations. @ 2012 American Institute of Physics. [http://dx.doi.org/10.1063/1.3685721]
\end{abstract}

\section{INTRODUCTION}

A fluid at rest can be set into motion if it is subjected to a difference in temperature (thermoconvection) or to a difference in electric potential (electroconvection). Even if the mechanisms are totally different, the results are similar: the liquid moves and some typical patterns of flow (rolls, hexagons, etc.) can be observed in both cases. The electroconvective phenomenon with a dielectric liquid placed between two infinite parallel metallic electrodes (one of them being at a high potential and the other earthed) is well known and has already been extensively investigated over the last few decades. It is also well known that the motion of the fluid is the consequence of the action of the electric field on the electric charges injected in the liquid at the interface between the liquid and the electrodes. The electrochemical mechanisms giving birth to the injection of charges at the electrodes have also been studied in depth, most notably by Felici. ${ }^{1}$ The injection of electric charges is a phenomenon generated by positive or negative electrodes when immersed in liquids of low enough conductivity, and when the electric field applied is sufficiently high. It can take place at both electrodes or at only one of them. In our article, we consider that the injection occurs at one electrode (unipolar injection) only. The non-dimensional electrical parameter $C=\frac{q_{0} H^{2}}{\varepsilon \Delta V}$, which measures the level of injection (weak when $C$ is lower than 0.1 , strong when $C$ is greater than 10), plays an important role in the phenomenon. It depends on the space charge density at the electrode $q_{0}$, the height $H$ of the layer, the electric permittivity $\varepsilon$ of the liquid, and the applied voltage $\Delta V$. 
The motion of the fluid starts with unstable rolls, which degenerate very quickly into hexagons. In 1969, Felici $^{2}$ proposed a simple hydraulic model for the roll electroconvective cells, neglecting the inertial effects and considering the case of weak unipolar injection. He modelled the rolls by two columns of the same radius, one for the liquid moving up and another for the liquid moving down (the layer is supposed to be horizontal) with a constant velocity profile. His model explained the onset and the persistence of the steady finite amplitude motion of the liquid, in qualitative terms, in an initially static plane layer of dielectric liquid. This type of flow is governed by a characteristic dimensionless number referred to as $T$ that represents the ratio of the destabilizing Coulomb force to the stabilizing viscous one. The author established the existence of a linear stability criterion $T_{c}$ associated with the growth of infinitely small amplitude perturbations. The existence of another stability criterion $T_{f}\left(T_{f}<T_{c}\right)$ corresponding to finite amplitude velocity disturbances was also highlighted. Later, Atten and Lacroix ${ }^{3}$ extended Felici's hydraulic model to the case of hexagons. They developed a nonlinear analysis based on the Galerkin method, and also predicted the existence of a nonlinear stability criterion lower than the linear one. The results of their experiments for different dielectric liquids are in agreement with their nonlinear stability theory (existence of two criteria) and the emergence of hexagonal patterns, even if there is a discrepancy between the calculated and the measured values for the onset of the electrohydrodynamics (EHD) instability which has not yet been explained.

Many works have been carried out to examine the stability of the electroconvective phenomena. The first attempt to numerically solve the whole set of equations was made by Castellanos, Atten, and Perez. ${ }^{4}$

They used the finite difference method and SIMPLER (Ref. 5) algorithm to solve the momentum equations, as well as the Poisson equation for the potential and the charge density conservation equation. They indicated that the strong numerical diffusion of the scheme invalidated the method, especially looking at results concerning time-dependent convection and nonlinear criterion. It is well acknowledged from a strict numerical point of view that the way the charge density equation is solved, due to its hyperbolic nature and the existence of sharp charge gradients, is the critical issue in such electroconvective problems. ${ }^{6}$ This is why, in an alternative method, they "numerically" inject a finite number of charged particles to simulate the effects of true ions in the fluid, instead of integrating the charge density equation. These charged particles are displaced by the fluid velocity field and the electric field. But, contrary to their previous attempt, this time the velocity field was not computed from the Navier-Stokes equations. The velocity field is constrained to follow an analytical form from a given stream function, which is supposed to reproduce the flow structure with an amplitude factor that must be computed. The amplitude of the velocity roll is determined from the balance between the electric and viscous forces. The drawback of this kind of approach is that the velocity field, as well as the electrical field, are not really computed from equations but given a priori. This method to solve the charge density equation belongs to the so-called super-particle methods, and it has been extensively investigated by other authors, among them, Chicón et al. ${ }^{7,8}$ Significant improvements were made in their papers. They still applied the super-particle method to compute the charge density field, but this time they entirely solved the potential from Poisson's equation in order to compute the electric field. Nevertheless, the fluid velocity field was still not computed from the Navier-Stokes equations, and the same method as in Ref. 4, using the amplitude and stream function, was employed.

Quite recently, Vázquez et al. ${ }^{9}$ presented an excellent comparison between the two available methods for solving the charge density: the super-particle method and the integration in time of this equation. To accomplish this, they choose a finite element method associated with the FCT scheme (flux corrected transport, Boris et al. ${ }^{10}$ Zalesak $^{11}$ ). As their predecessors, the fluid velocity field is not still computed with the Navier-Stokes equations, but imposed by a given stream function with a variable amplitude. The most obvious conclusion is that the super-particle method is less diffusive than the FCT scheme and, hence, produces better results than its counterpart. However, small oscillations in the velocity field and, consequently, in the electric field, which were found in the case of super-particle method that were absent with the FCT scheme. According to Vázquez et al., ${ }^{9}$ the main drawback of super-particle method compared to a commonly used scheme such as FCT is definitely its cost in terms of CPU time. 
Another attempt to solve the entire set of coupled equations can be attributed to Tsai et al. ${ }^{12}$ for their study of electroconvection in an annular film. They used a pseudo-spectral method with Chebyshev polynomials in the radial direction and azimuthal direction to compute the velocity field from the Navier-Stokes equations which are considered in stream-vorticity formulation. All the other relevant variables for this problem are also approximated by truncated Fourier series and Chebyshev polynomials.

In this paper, we also numerically solve the whole set of coupled equations associated with the phenomenon of electroconvection between two plane electrodes, using the same method we developed in Ref. 13. No assumptions are made for the fluid velocity field, and the Navier-Stokes equations in primary variables $(\vec{u}, p)$ including Coulomb force are solved using a finite volume technique $^{5}$ associated with the Augmented Lagrangian and Uzawa algorithms for velocity-pressure coupling. ${ }^{14,15}$ When the velocity field is accurately computed from the Navier-Stokes equations, the charge density transport equation is then integrated using the SMART algorithm (sharp and monotonic algorithm for real transport, Gaskel et al. ${ }^{16,17}$ ) in order to efficiently account for its hyperbolic nature without excessive numerical diffusion, oscillations free and boundedness criteria. In Ref. 18, it has been demonstrated that this scheme was less diffusive than the FCT scheme. Then in sequence, the Poisson equation for potential is solved and the electric field can be computed from it.

The remainder of this paper is organised as follows. In Sec. II, the problem is stated and we provide the set of non-dimensional equations associated with the electroconvective problem of a horizontal liquid layer subjected to unipolar injection. We define the relevant dimensionless parameters and we present the numerical method derived to solve the whole set of the coupled governing equations. The following section (Sec. IV) presents various results starting with a comparison between numerical and analytical profiles of charge density in the hydrostatic case. The structure of this electroconvection flow is finely detailed for the case of strong injection. The route to the chaos is analysed from the pure steady laminar state until the fully chaotic regime. Several other comparisons are made about the linear and nonlinear criteria obtained with our direct numerical simulations and those obtained with a stability approach.

Furthermore, we provide the variation of the electric Nusselt number in terms of the nondimensional electric parameter $T=\frac{\varepsilon \Delta V}{K \eta}$, where $K$ is the mobility of the ions and $\eta$ is the dynamic viscosity of the fluid. A spectral analysis is then conducted which relates the frequency and power spectra to the main parameters in different simulations. Finally, a conclusion is drawn in Sec. V.

\section{FORMULATION OF THE PROBLEM}

\section{A. Basic governing equations}

The system under consideration in this article is a dielectric liquid layer of width $H$ enclosed between two electrodes of length $L$ (Figure 1). The layer is subjected to a potential difference $\Delta V=V_{0}-V_{1}$. The z-axis is taken as perpendicular to the electrodes. Electric charges are injected from electrode 0 .

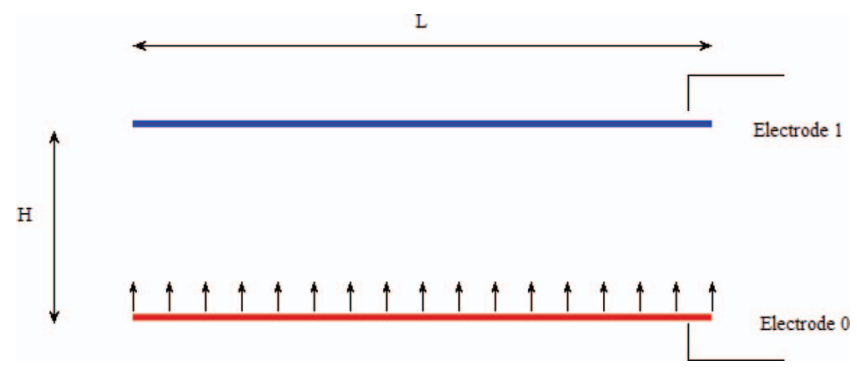

FIG. 1. (Color online) Sketch of the physical domain. 
The complete characterisation of the flow of dielectric liquids subjected to electric fields is governed by the EHD equations (see, for example, Refs. 19-21). For the case of an isothermal electrically insulating liquid of density $\rho_{0}$, dynamic viscosity $\eta$, and permittivity $\varepsilon$, confined between perfectly conducting parallel plates of distance $H$ apart and subjected to pure unipolar injection, these equations reduce to

$$
\vec{E}=-\nabla V
$$

The Gauss law

$$
\nabla \cdot(\varepsilon \vec{E})=q
$$

The conservation of charge density

$$
\frac{\partial q}{\partial t}+\nabla \cdot \vec{j}=0
$$

The Navier-Stokes equations

$$
\rho_{0}\left(\frac{\partial \vec{u}}{\partial t}+(\vec{u} \cdot \nabla) \vec{u}\right)=-\nabla p+\eta \Delta \vec{u}+\vec{f}_{e},
$$

and the continuity equation

$$
\nabla \cdot \vec{u}=0,
$$

where $q$ is the charge density, $\vec{j}$ is the electrical current density, $\vec{u}$ is the fluid velocity, and $p$ is the pressure. $\vec{f}_{e}$ is the density of the electric force whose expression can be found in Ref. 22

$$
\vec{f}_{e}=q \vec{E}-\frac{E^{2}}{2} \nabla \varepsilon+\nabla\left[\frac{E^{2}}{2} \rho \frac{\partial \varepsilon}{\partial \rho}\right] .
$$

In an isothermal and homogeneous fluid $\nabla \varepsilon=0$, so the second term of the electric force that is the dielectric force vanishes. By including a third term: the electrostriction force in the pressure gradient term $p+\left[\frac{E^{2}}{2} \rho \frac{\partial \varepsilon}{\partial \rho}\right]=\tilde{p}$, Eq. (4) simplifies to

$$
\rho_{0}\left(\frac{\partial \vec{u}}{\partial t}+(\vec{u} \cdot \nabla) \vec{u}\right)=-\nabla \tilde{p}+\eta \Delta \vec{u}+q \vec{E} .
$$

Assuming a linear isotropic medium and the four classical distinct mechanisms of the charge transfer: convection, migration, diffusion, and conduction, we may write the constitutive equation for the current density

$$
\vec{j}=q \vec{u}+q K \vec{E}-D \nabla q+\sigma \vec{E},
$$

where $K, D$, and $\sigma$ are, respectively, the ionic mobility, charge-diffusion coefficient and the conductivity. It should be noted that the magnetic effects as well as Joule heating are fully negligible since the currents generally observed, in these assumed perfectly insulating liquids, are very weak. For the same reason in this problem the conductivity $\sigma=0$. With the diffusion current also being neglected, there remain only two components in the current density: the convection current $q \vec{u}$ associated with the convection of charge by the fluid velocity and the migration current $q K \vec{E}$ due to the drift velocity of ions.

In these circumstances, the charge density conservation equation (3) simplifies to

$$
\frac{\partial q}{\partial t}+\nabla \cdot(q(\vec{u}+K \vec{E}))=0 .
$$

From a physical point of view, it is necessary to know in detail the physicochemical processes close to the electrodes to obtain injection and ejection laws relating charge density to electric field strength. These laws would specify the way in which the charge is injected into the fluid at the emitter electrode and removed at the collector one. In order to avoid the difficulties associated with a discussion of the complex electronic processes occurring at the electrode's surfaces, we assumed that we have a homogeneous and autonomous injection of unipolar charge at the emitter electrode. 
That means that $q=q_{0}$ at $z=0$ at all times, i.e., the injector, and hence the injection rate, are neither influenced by the electric field nor by the liquid motion. A more realistic alternative exists which consists of defining some injection laws which links the injection rate to the electric field. A revision of the different injection models can be found in Ref. 23 .

However, it is worth noting that the effect of using a more realistic injection law would be to produce, at most, a charge-diffusive boundary layer at the electrode without affecting the mainstream solution of our problem.

\section{B. Dimensionless parameters and equations}

For universality in the description of the problem, it is particularly convenient to work with non-dimensional equations. In order to transform the last set of equations into a non-dimensional one, we introduce the following dimensionless quantities, denoted with a star:

$$
x_{i}^{*}=\frac{x_{i}}{H}, \rho^{*}=\frac{\rho}{\rho_{0}} u_{i}^{*}=\frac{u_{i}}{u_{0}}, q^{*}=\frac{q}{q_{0}} p^{*}=\frac{p}{\rho_{0} u_{0}^{2}}, V^{*}=\frac{V}{\left(V_{0}-V_{1}\right)}, E_{i}^{*}=\frac{E_{i}}{\left(V_{0}-V_{1}\right) / H} .
$$

This leads to the following set of dimensionless parameters:

$T=\frac{\varepsilon \Delta V}{\rho_{0} \nu K}$ which is the ratio of Coulomb and viscous forces.

$C=\frac{q_{0} H^{2}}{\varepsilon \Delta V}$ is the dimensionless measure of the injection level.

$M=\frac{1}{K}\left(\frac{\varepsilon}{\rho_{0}}\right)^{1 / 2}$ is the ratio between the so-called hydrodynamic mobility and the true mobility of ions.

Several choices for the reference velocity $u_{0}$ are possible. In this study, the most obvious choice could be $u_{0}=\frac{K\left(V_{0}-V_{1}\right)}{H}$ using the ionic mobility $K$. For convenience, we shall also use $R=\frac{T}{M^{2}}$ which is defined as the electrical Reynolds number.

If we drop the star indicator for clarity, the set of non-dimensional equations becomes

$$
\begin{gathered}
\nabla \cdot \vec{u}=0 \\
\frac{\partial \vec{u}}{\partial t}+(\vec{u} \cdot \nabla) \vec{u}=-\nabla p+\frac{1}{R} \Delta \vec{u}+C M^{2} q \vec{E} \\
\frac{\partial q}{\partial t}+\nabla \cdot(q(\vec{u}+\vec{E}))=0, \\
\Delta V=-C q, \\
\vec{E}=-\nabla V .
\end{gathered}
$$

A more comprehensive description of the equations and the parameters involved can be found in Refs. 22 and 23.

\section{Initial and boundary conditions}

As our set of equations is time dependent, one has to provide initial conditions. As a general rule, we start either from the fluid at rest or from a steady state obtained from a previous simulation. For the boundary conditions (Figure 2), two different cases have been considered.

\section{Rigid walls case}

On each wall, a no-slip boundary condition has been imposed: $u=v=0$. 


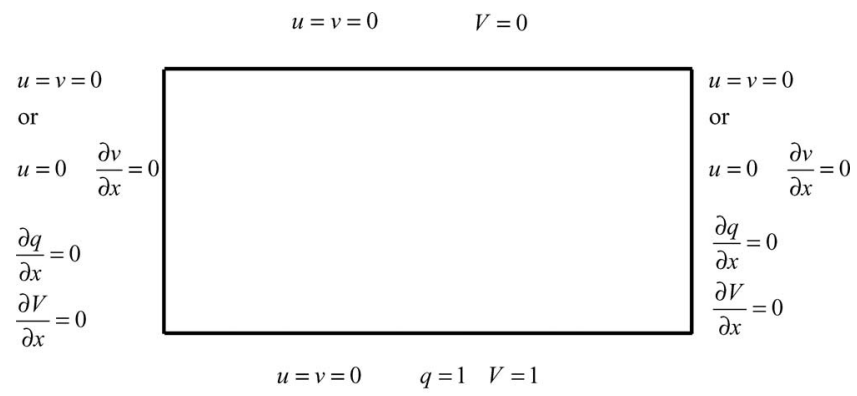

FIG. 2. Computational domain and boundary conditions.

\section{Symmetric boundary case}

In this case, symmetrical boundary conditions for velocity have been considered on the lateral borders in order to simulate an infinitely long cavity: $u=0 \quad \frac{\partial v}{\partial x}=0$.

\section{NUMERICAL METHOD}

\section{A. Discretization practice}

The problem under consideration is mathematically described by the conservation equations for mass, momentum, and charge density, and by Poisson's equation for potential. The above set of coupled partial differential equations (Eqs. (6)-(10)) is discretized using a finite-volume approach on a staggered grid. All the details on the finite-volume discretization methods used here are given by Patankar. ${ }^{5}$ Only general outlines are given in this section with an emphasis on the treatment of the charge density transport equation.

\section{B. Charge density equation treatment}

Since steep gradients may appear, considering the hyperbolic nature of the transport equation for charge density $q$, the well-known central difference scheme will introduce undesirable over and under shoots, while the upwind difference scheme will smear the solution in an unacceptable way.

To prevent the development of possible oscillations of the solution, due to the lack of physical diffusion in the charge density transport equation, it is recommended adding artificial or numerical viscosity and also using non-oscillating, non-diffusive, and bounded schemes (Godunov ${ }^{24}$ ). Any other scheme that does not have these properties would lead to an oscillating or extra diffusive solution and to the failure of the solution process.

Many schemes among which essentially non-oscillatory schemes, FCT, ${ }^{10,11}$ MUSCL (Monotone Upstream Centered Schemes for Conservation Laws), ${ }^{25-27}$ Lax Wendroff, ${ }^{28}$ Davis, ${ }^{29}$ and Roe, ${ }^{30}$ can avoid these undesirable behaviours. The main desirable and strongest property for these schemes is total variation diminishing (TVD) (Refs. 31 and 32).

The purpose of such schemes is to provide an expression for $q_{k}$ on each face $S_{k}$ of the control volume to compute the convective flux $F_{k}^{C}=\dot{m}_{k} q_{k}$ through that face. Here, $\dot{m}_{k}$ stands for the mass flux through the same face. The idea is to add an additional flux contribution $\frac{\dot{m_{k}}}{2}\left(q_{D}-q_{C}\right) \psi\left(r_{k}\right)$ to the upwind convective flux $\dot{m}_{k} q_{C}$ to improve the order of accuracy and to limit the smearing of the solution. Sweby ${ }^{32}$ provides the requirements for second-order accuracy. This function $\psi$, called the limiter function, must also be chosen to suppress the possible wiggles appearing when high-order schemes are used. In this context, it has been established that the main property of that function is monotonicity preserving in order to prevent the creation of new undershoots and/or overshoots in the solution. The TVD property assures that monotonicity is preserved (Lien et al. ${ }^{33}$ ). 


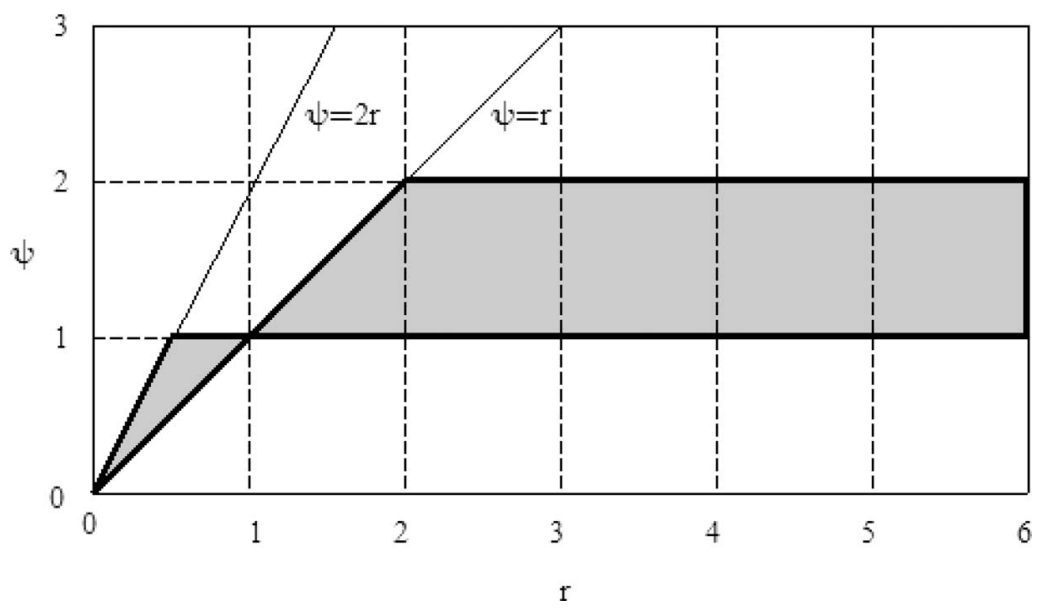

FIG. 3. Admissible region for a second order TVD scheme

The shaded region of the $\psi-r$ diagram in Figure 3 indicates the location where the so-called limiter function $\psi$ must be defined in order to constrain the range of possible values of the additional convective flux introduced in the second-order TVD scheme.

These kinds of schemes may be presented in an unified way by using the concept of upwindbiased expressions (Waterson et al. $\left.{ }^{34}\right): q_{k}=q_{C}+\frac{1}{2} \psi\left(r_{k}\right)\left(q_{D}-q_{C}\right.$ ), where $r_{k}=\frac{q_{C}-q_{U}}{q_{D}-q_{C}}$ is the local ratio of upstream gradient to downstream gradient expressed at face centre $k, q_{C}$ the mean central node, $q_{U}$ the mean upstream node, and $q_{D}$ the mean downstream node (see Figure 4).

The convective flux on face $k$ is expressed as: $F_{k}^{C}=\dot{m}_{k} q_{C}+\frac{\dot{m_{k}}}{2} \psi\left(r_{k}\right)\left(q_{D}-q_{C}\right)$. The first part of this expression will be treated implicitly, while the second part will be reported on the right-hand side and treated explicitly using the deferred correction approach of Koshla and Rubin. ${ }^{35}$ We have chosen the second-order SMART scheme. ${ }^{16,17}$ From our experience, it perfectly fits the requirements needed for an accurate charge density solution. In this scheme, the limiter function is given by: $\psi(r)$ $=\max (0, \min (2 r, 0.75 r+0.25,4))$. Several very classical tests (advection of an initial condition: step profile in $1 \mathrm{D}$, advection and rotation of a square or a cone shaped in $2 \mathrm{D}$ ) have been performed to validate successfully the solver.

\section{RESULTS AND DISCUSSIONS}

In this paper, we study the strong injection case for which $C$ is taken to be 10 . The code has been validated through several comparisons between numerical results and theoretical ones, when they are available, or by the results of linear stability analysis. ${ }^{13}$ The accuracy of the numerical method presented in Sec. III is carefully analysed through various grid convergence checks.
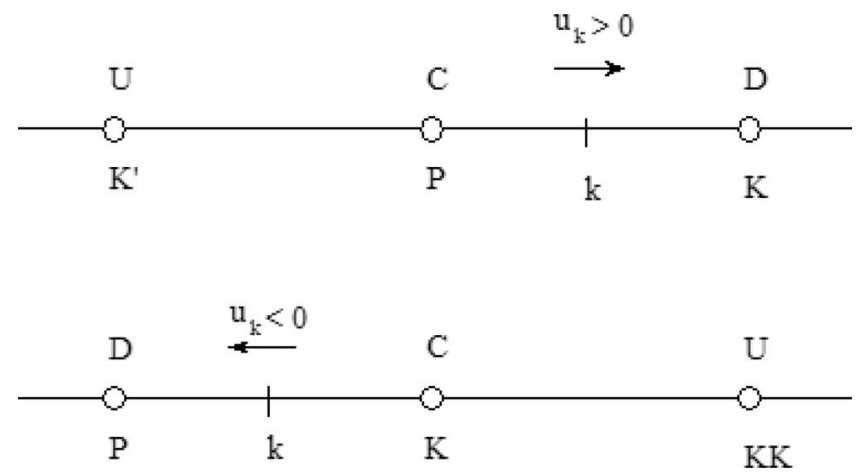

FIG. 4. Localisation of nodes $\mathrm{C}, \mathrm{D}$, and $\mathrm{U}$ towards $k$ and sign of velocity in 1D. 


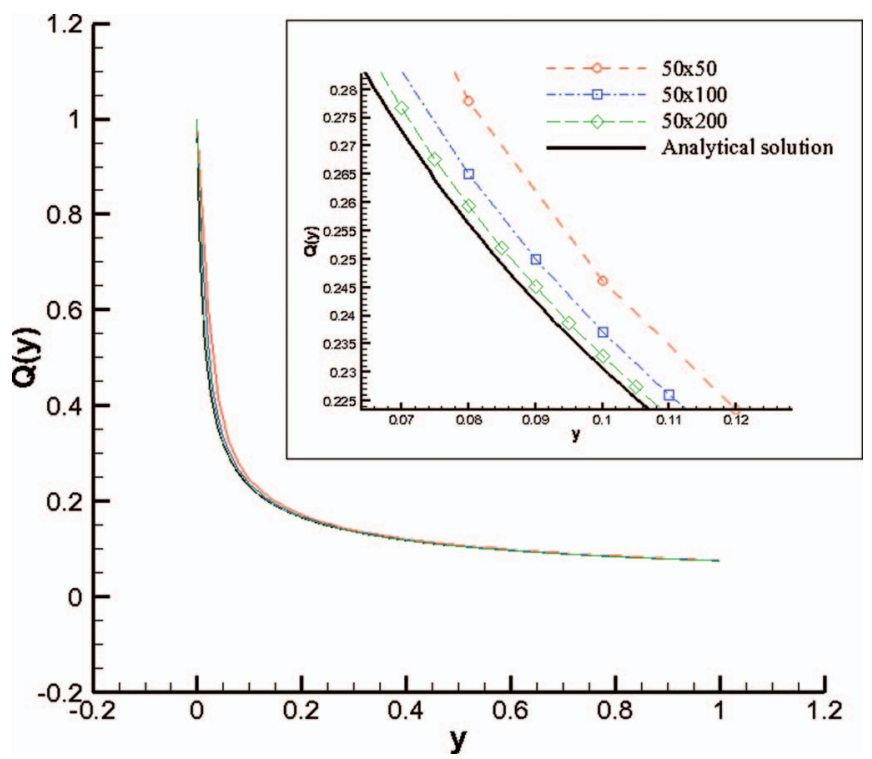

FIG. 5. (Color online) Charge density profile corresponding to the analytical steady hydrostatic solution. $L=0.614$, $T=100, M=10, C=10$.

In Figure 5, we have plotted the comparison between the analytical profiles of charge density obtained in the case of a steady hydrostatic solution for various grid sizes: $50 \times 50,50 \times 100$, and $50 \times 200$. This hydrostatic solution corresponds to the analytical solution in the case where the fluid velocity is taken as zero.

This solution is given by $Q(z)=3 \cdot / \sqrt{\left(9+16 C^{2} z\right.}$.

In practice, the code is run for a value of $T$ below the critical value $T_{c}$ for which the instability starts to grow. In that case, whatever the initial conditions, the fluid velocity will tend to zero converging towards the steady hydrostatic solution. A perfect agreement is found even for the coarsest grid of $50 \times 50$.

\section{A. Linear criterion}

The linear stability analysis (Schneider et al. ${ }^{36}$ and Atten et $a l .{ }^{37}$ ) usually considers the periodic perturbation of an infinite fluid layer. Therefore, in order to numerically study the linear stability problem, we have applied symmetrical boundary conditions to the lateral bounds. The perturbations of every physical quantity $f$ grow close to the instability threshold following an exponential law $f=f_{0} e^{\sigma t}$. The growth rate $\sigma$ is positive above the instability threshold and negative below it. The linear instability threshold can be accurately computed from the growth rate of linear perturbations for different values of the parameter $T$.

We have used the maximum value of the velocity $V_{\max }$ in the domain of computation as a representative physical quantity. It is expected that, in the very first states of growth, the velocity follows the law $v_{\max }=v_{0} \exp (\sigma t)$.

We let the system evolve from a rest state. The tiny numerical fluctuations always present are sufficient to induce motion. After an initial period of latency in which the velocity is very small and varies erratically, typically some ten units of non-dimensional time, a first interval of exponential growth is observed. Following this first period, a second interval of greater growth rate is observed. We have computed the growth rate corresponding to the first interval, which corresponds to the slope of the straight line given by: $\log \left(V_{\max }\right)$ versus $t$ in this first interval (see Figure 6(b)). Since in this 


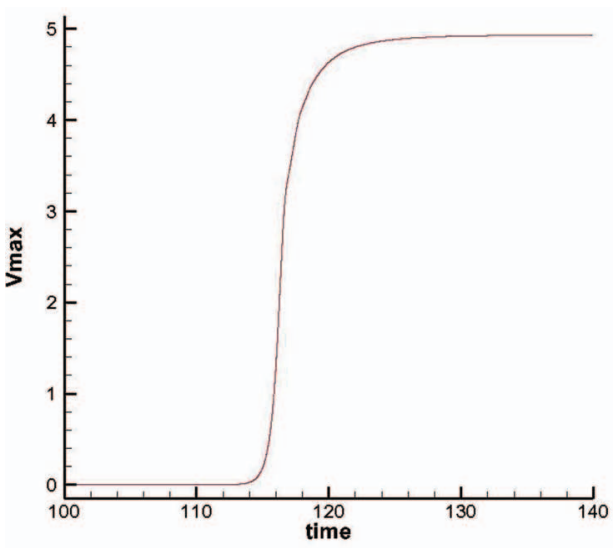

(a) Vmax vs time

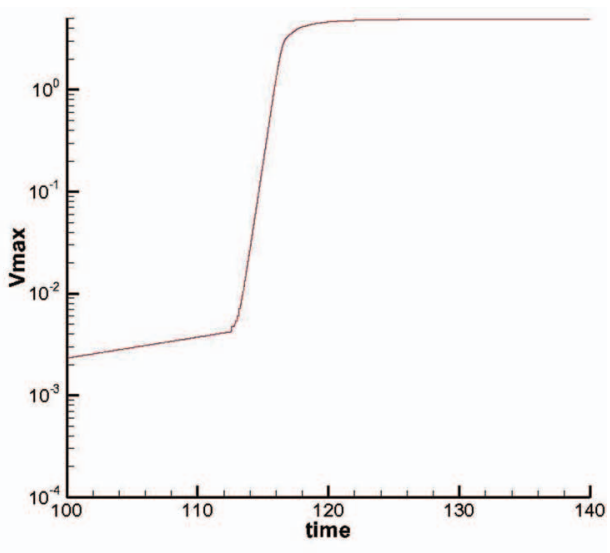

(b) Vmax vs time (semi-log plot)

FIG. 6. (Color online) Time evolution of the maximum velocity in the domain from initial state until steady state. $L=0.614$, $T=250, M=10, C=10$. (a) $V_{\max } v s$ time; (b) $V_{\max } v s$ time (semi-log plot).

interval the velocity is smaller than in the second one, this growth rate is more likely to be related to the linear regime.

Very close to the critical value, the growth rate $\sigma$ is a linear function of $T$, but in our numerical conditions case $T \in[175,250]$ a parabolic fitting of the curve $\sigma(T)$ is more appropriate. The critical value $T_{c}$ is obtained for $\sigma=0$ as shown in Figure 7. The critical value for $L=0.614$ corresponding to a half wavelength, has been found equal to 155.64.

As a test of the adequacy of the symmetrical boundary condition, we have computed the growth rate at the same interval of $T$ for $L=10$. For this value of $L$, the system is less constraint to choose the size of the convective cell. Figure 8 shows that in this case the results are very similar, independent of the nature of the boundary conditions on the lateral walls, symmetric, or rigid boundary conditions. The critical values in the both cases are 155.9 for the symmetrical boundary case and 155.67 in the rigid case, very close to that found in the case of $L=0.614$ (155.64).

Figure 9 shows the variation of $T_{c}$ with the horizontal extension of the computational domain $L$. Symmetrical boundary conditions have been applied in these computations to the lateral walls. The first minimum is found for $L=0.614$ and $T=155.64$, to be compared with the results obtained by

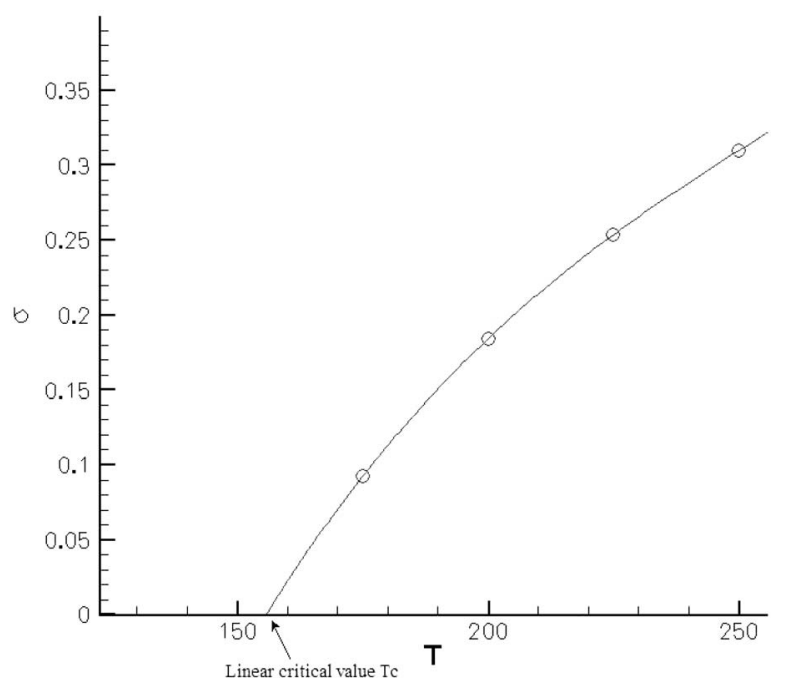

FIG. 7. Growth rate $\sigma$ versus $T$ for $L=0.614$ and $M=10$. Symmetrical boundary conditions have been applied to the lateral walls. 


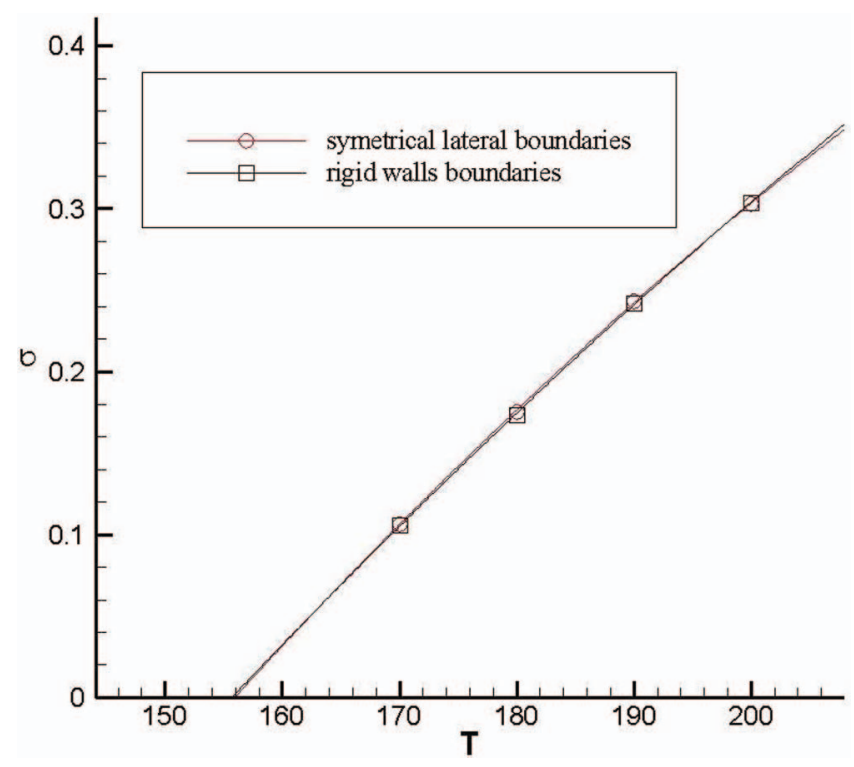

FIG. 8. (Color online) Growth rate $\sigma$ versus $T$ for $L=10$ for the symmetrical boundary and rigid walls cases.

Atten et al. ${ }^{37}$ of $L=0.614, T=164.1$ from the linear analysis. The successive minima correspond to the same value of $T$ and multiples of $L=0.614$. They occur at multiple numbers of the most unstable convection cell because in that case a multiple number of convection cells of size $L=0.614$ is possible. The existence of maxima is also associated with the symmetrical boundary conditions. In effect, when $L$ approaches the value 0.9 , the value of $T$ associated with two convective cells of $L$ $=0.45$ is smaller than that associated with a single convective cell of $L=0.9$. Therefore, the system naturally evolves towards two convective cells. The maximum near $L=1.5$ is even smaller, because in that case $L=0.5$ has a value of $T$ smaller than $L=0.75$, and the system evolves towards three convective cells. In conclusion, the curve $T(L)$ has minima every multiple of $L=0.61$ with the same value of $T$, and maxima between two successive minima that are smaller as $L$ increases.

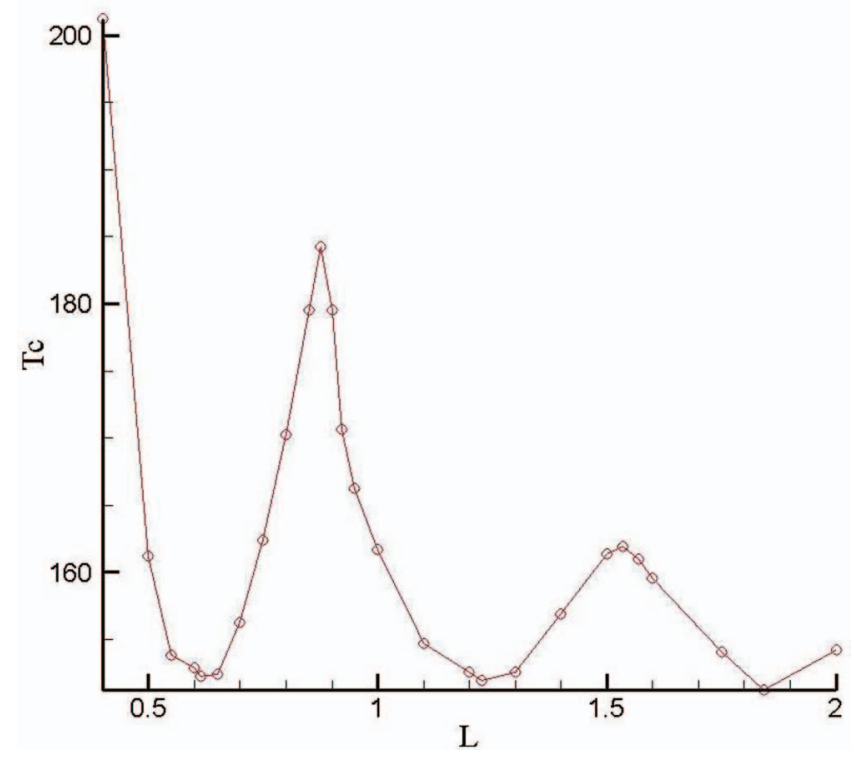

FIG. 9. (Color online) $T_{c}$ versus $L$ for $C=10$ and $M=10$. Symmetrical boundary conditions have been applied to the lateral walls. 


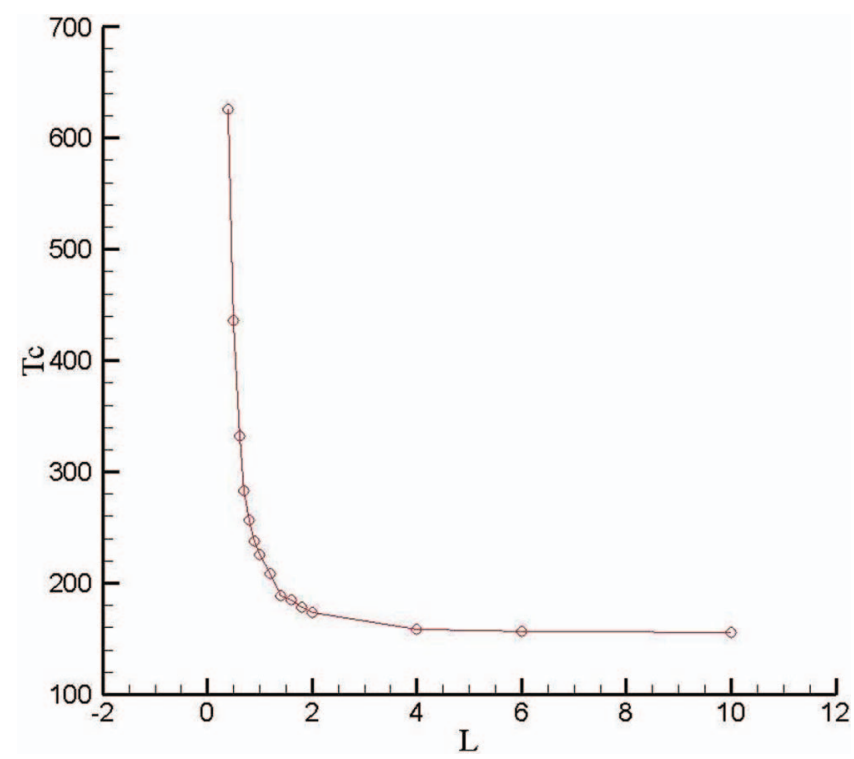

FIG. 10. (Color online) $T_{c}$ versus $L$ for $C=10$ and $M=10$. The boundary conditions applied to the lateral borders correspond to the rigid walls.

If, instead of symmetrical boundary conditions, we consider that the lateral walls are rigid, these periodic minima disappear. As Figure 10 shows, the linear criterion $T_{c}$ is a decreasing function of the domain length $L$ when no-slip boundary conditions are applied to the lateral walls, since less and less constraint is placed on the motion for increasing values of $L$. In this case, we expect that for $L \rightarrow \infty$ the critical value of $T$ approaches the value obtained from the linear analysis for a layer of infinite lateral extension. The observed deviations of the expected behavior are due to the fact that when the computational domain is increased, more points are needed to maintain the same computational accuracy.

\section{B. Nonlinear criterion}

Felici ${ }^{2}$ and Atten and Lacroix ${ }^{3}$ showed that, above the linear criterion, a convective regime is established in which the maximum velocity is greater than the ion drift velocity $K\|\vec{E}\|$, (in our non-dimensional units). Once the convective regime is developed, decreasing the value of $T$ below the linear instability threshold does not result in the suppression of motion until a second value, the nonlinear criterion, is reached. Therefore, a hysteresis loop is associated with these two values of the stability parameter $T$.

It is more difficult to obtain a precise determination of the parameter $T$ for nonlinear criterion than for the linear one.

In order to determine the nonlinear criteria, we have used the following procedure. Starting from a rest state, we have run the code for a value of $T$ above the linear criterion. The velocity increases until a new steady state of finite amplitude is obtained, as is shown in Figure 17. The maximum velocity for this value of $T$ is noted. Taking the velocity, pressure, charge, and potential distributions of this last simulation as the initial conditions, we decrease the value of $T$ and run the code again until a steady state is reached once more, but corresponding to the new value of $T$. Further decreasing $T$, we compute the curve $v_{\max }$ versus $T$. For a certain value of $T$, the velocity reduces to zero. This second value is referred to as the nonlinear criterion $T_{f}$ and corresponds to the stability limit of the rest state to nonlinear perturbations. In the case $L=0.614, M=10$, and $C=10$, we found $T_{f}=107.5$ which is in a good agreement with the predicted value of 110 given by the nonlinear stability analysis of Atten and Lacroix. ${ }^{3}$ The overall hysteretic behaviour is depicted in Figure 11. 


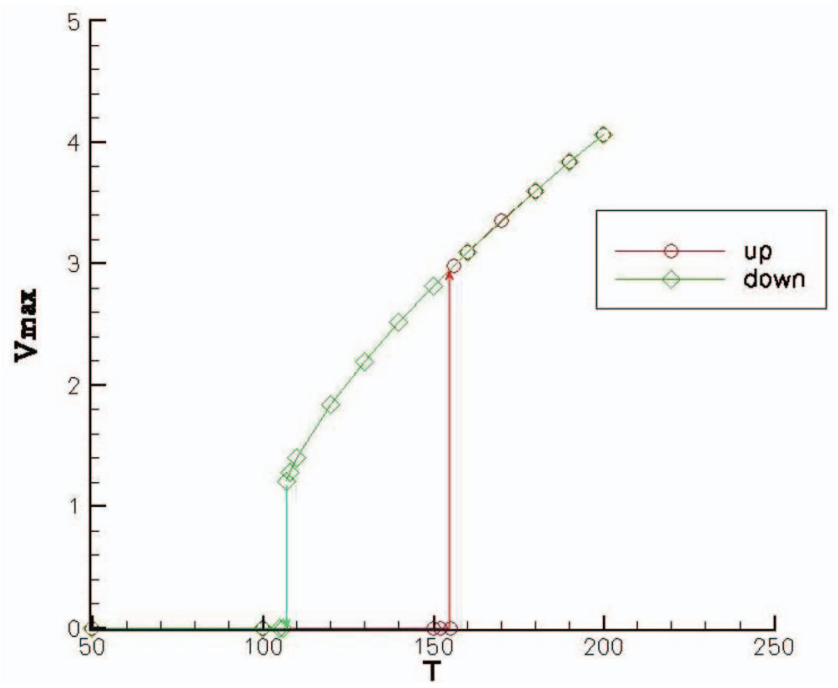

FIG. 11. (Color online) Evolution of $V_{\max }$ versus $T$ for $C=10, L=0.614$, and $M=10$. Symmetrical boundary conditions have been applied to the lateral walls.

This hysteresis loop is similar to the one obtained by Figure 10 of Chicón et al. ${ }^{7}$ In particular, the value of the velocity amplitude at the linear criterion is close to 3 . The hysteresis loop for lateral walls is indicated in Figure 12(a). This figure is similar to Figure 12(b), the only difference being the values of the linear and nonlinear criterion that are greater than those for symmetrical boundary conditions. This is physically sounded, since the rigid walls present a greater resistance to liquid motion. Also, the values of the maximum velocity for a given value of $T$ are smaller than in the symmetrical boundary conditions case, for the same reasons.

\section{Finite amplitude convection}

As we have stated, the linear stability analysis indicates that the half wavelength of the most unstable mode in the case of strong injection is $\lambda=0.614$ (Atten et al. ${ }^{37}$ ). The number of convective cells in a domain of length $L, N_{\text {cell }}$, is directly linked to the values of $\lambda$ and $L$ as it is the closest integer

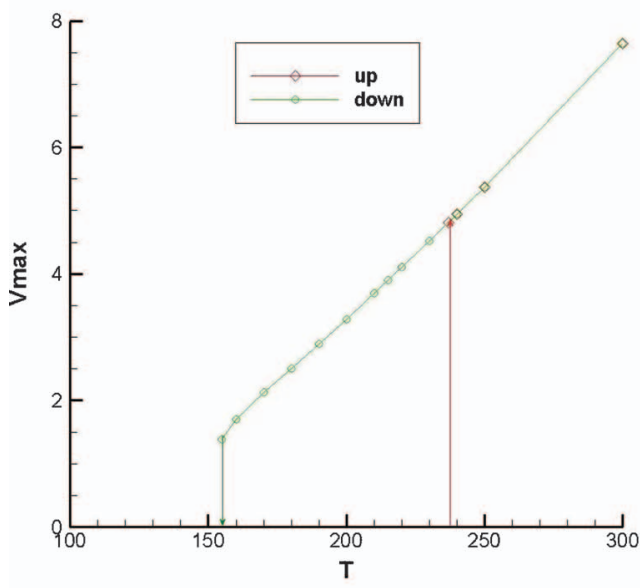

(a) rigid walls

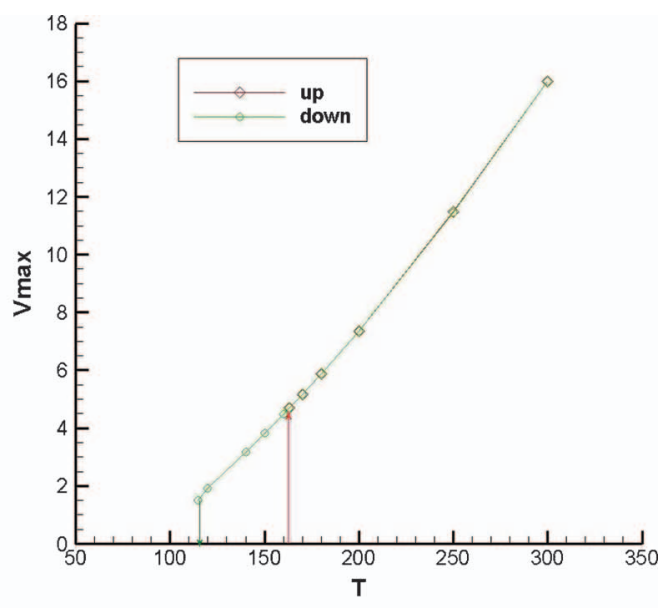

(b) symmetric boundary

FIG. 12. (Color online) Evolution of $V_{\max }$ versus $T$ for $C=10, L=1$, and $M=10$. (a) Rigid walls; (b) symmetric boundary. 

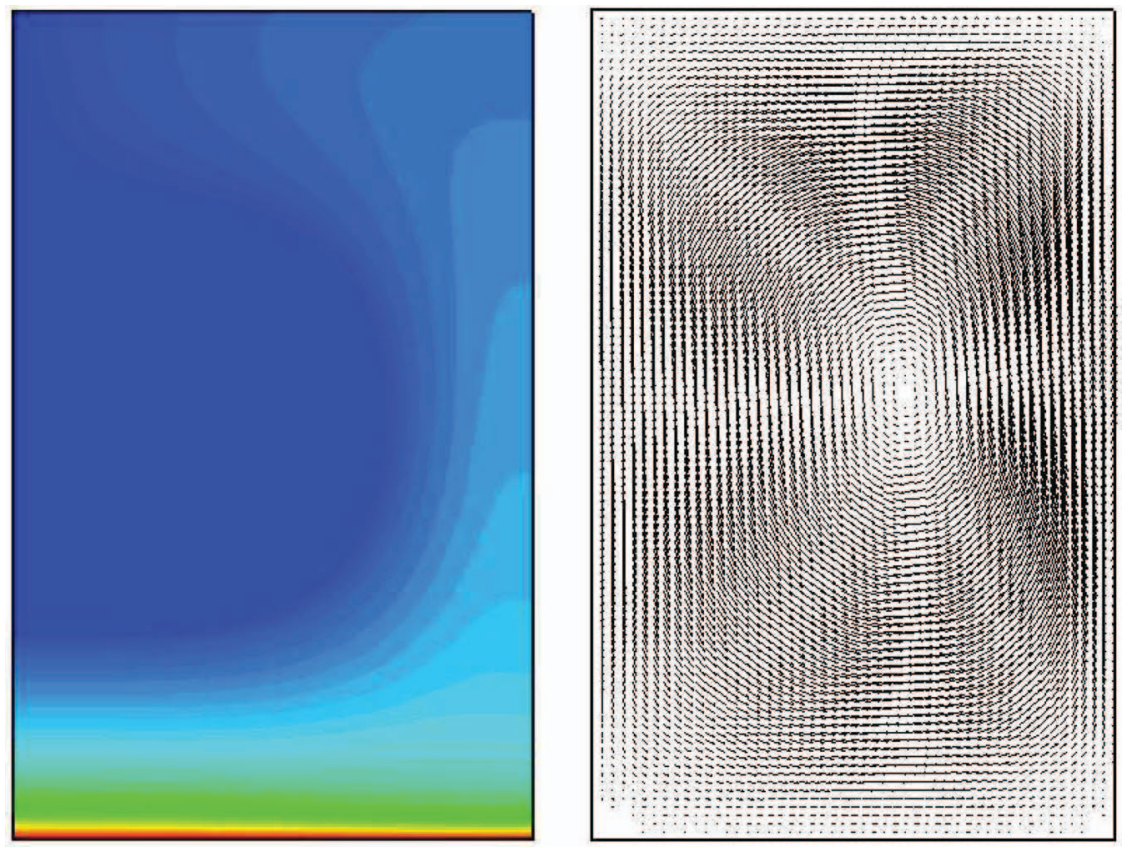

FIG. 13. (Color online) Charge density isocontours and velocity field. $L=0.614, T=175, M=10, C=10$.

value of the ratio $\frac{L}{\lambda}$. This is strictly true for stationary regimes, but things change if non-steady behaviour evolves.

The structure of the flow adopts the following scenario. For a value of $T<T_{c}$, no fluid motion is observed. Increasing $T$ just above the critical value of $T_{c}$, the fluid motion starts and one convective cell appears in the cavity. In Figure 13, we have presented the isocontours of charge density as well as the velocity field for $T=175$, which is above the critical value $T_{c}$. The length of the domain is $L=\lambda=0.614$. For such a value of $T$, a steady state is reached and one convection cell is found as suggested by the linear stability analysis. On the lateral boundaries of the domain, symmetrical boundary conditions have been implemented. This structure of the flow constitutes a good test case for validation. Indeed, according to Castellanos and Atten, ${ }^{38}$ above the critical value of the $T$ parameter when motion sets in, it always takes place with a maximum velocity greater than the ionic drift velocity and therefore a region develops where the convection cells are completely void of charge as indicated in Figure 13 and where ions cannot enter. The structure of the charge density contours with the region void of charge is a very important feature of electroconvection problems and numerical simulations should predict this behaviour.

Even if the determination of the linear and nonlinear critical values of the $T$ parameter predicted by our simulations is also a good indicator of the validity of our numerical method, these authors have shown that a finite difference method combined with a second-order upwind differencing scheme for the advection terms of the charge density transport equation were able and sufficient to track the critical value $T_{c}$ in the weak injection problem. However, this scheme was revealed to be inefficient when computing the charge distribution inside a convection cell. They believe, and we support the idea, that it is certainly due to the additional numerical diffusion induced by this scheme that tends to fill the void region. For the same reason, the velocity amplitudes for the hysteresis loop are well below those expected due to the underestimation of the electric torque in the cell. These drawbacks induced by extra numerical diffusion because of the inefficiency of the numerical scheme are well discussed and asserted in Pérez and Castellanos. ${ }^{6}$

When the domain is extended to ten times the initial half wavelength $\lambda$, we find as expected, and as should theoretically be the case according to the stability analysis, ten convection cells (see 

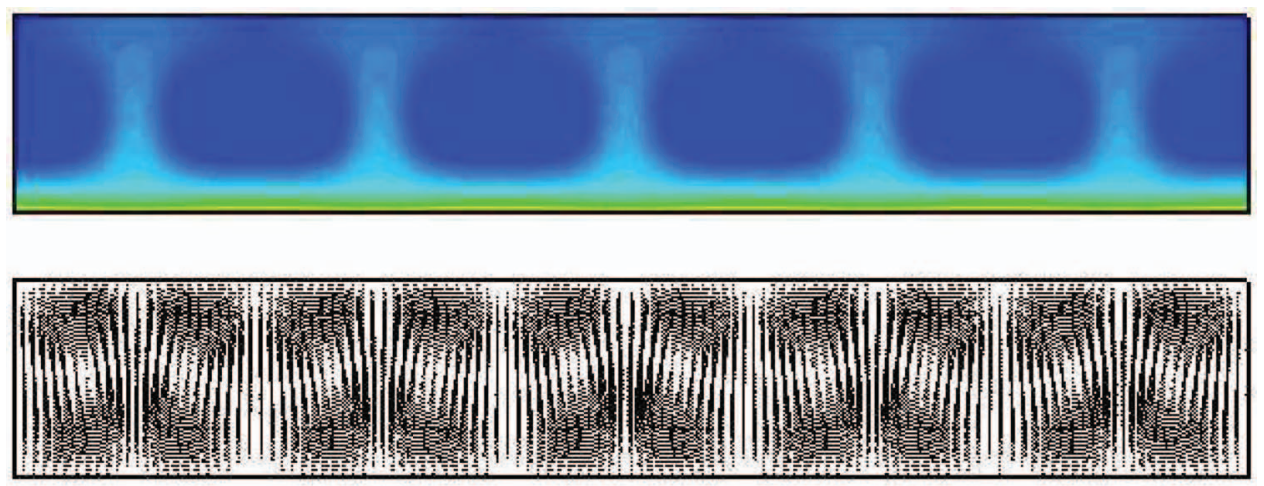

FIG. 14. (Color online) Charge density isocontours and velocity field. $L=10, \lambda=6.14, T=175, M=10, C=10$.

Figure 14). Our numerical method is completely consistent with the linear stability analysis for this regime.

\section{Flow structure for moderate values of $T$ parameter}

The tabs displayed in Figures 15 and 18 show several characteristic and patterns of the flow. Figure A plots the time history of the maximum velocity. Figure B represents the velocity field, while Figure $\mathrm{C}$ is a snapshot of the charge density isocontours to highlight the structure of the flow. Columns (a)-(c) are associated with the different $T$ values. The one convective cell structure is observed until $T$ reaches a value of 250 (see Figures $15 \mathrm{~B}$ (a) and $15 \mathrm{C}$ (a)). Even far from the critical $T$ value, the flow still maintains a laminar stationary regime. Above this characteristic value, the cell starts to oscillate periodically in time and space (see Figure $15 \mathrm{~A}(\mathrm{~b})$ ), anticipating a transition to more unsteady behaviour. Still increasing $T$, the structure turns from a one convective cell into two convective cells (see Figure $15 \mathrm{~B}(\mathrm{c})$ ). Figure $15 \mathrm{~A}(\mathrm{c})$ clearly indicates this transition through the temporal evolution of $V_{\max }$.

The transition from one cell to two cells is clearly visible in Figure 16, which shows the temporal evolution of the velocity field. Figure 17, which is an expansion of Figure 15 A(c), is the corresponding evolution of $V_{\max }$ versus time for the same simulation at $T=300$. After an exponential increase in the maximum of velocity, one reaches a state that seems to be steady for around 10 units of non-dimensional time. Then, this state slowly starts to oscillate before switching suddenly towards a new state corresponding to two steady cells. It is noticeable that once the two cells regime is reached, it remains stationary for a certain range of $T$ values. The flow keeps this configuration with two steady cells up to $T=500$.

We should point out that the change of flow structure, from the one cell to two cells pattern has also been observed by Vázquez et al..$^{39}$ with the particle in cell method (PIC). Figure $15 \mathrm{C}(\mathrm{c})$ should be compared with Figure 5 of Ref. 39. For them, the transition from one to two cells pattern occurs for $T=400$. But, if we consider that the linear instability threshold in their simulations is a slightly overestimated and also that they only performed computations for $T=100,200,300,400$, and 500, the value of $T=400$ is consistent with ours.

\section{Flow structure for high values of $T$ parameter and path for chaotic regime}

Above $T=500$, the two convective cells again start to oscillate slowly and the regime becomes unsteady but still periodic. This behaviour is depicted in Figure $18 \mathrm{~A}(\mathrm{a})$ for which $T=600$. As we increase $T$, the amplitude and the frequency of these oscillations also increase.

For higher values of $T$, above 1500, the unsteadiness of flow is more pronounced and a new regime characterised by the appearance of plumes arises (see Figure $18 \mathrm{~A}(\mathrm{~b})$ ). Still increasing $T$, the flow becomes fully unsteady (see Figure $18 \mathrm{~A}(\mathrm{c})$ ), and electroconvective plumes are generated more frequently in the flow as depicted in Figure $18 \mathrm{C}(\mathrm{c})$. As occurs in the Rayleigh-Bénard convection, 


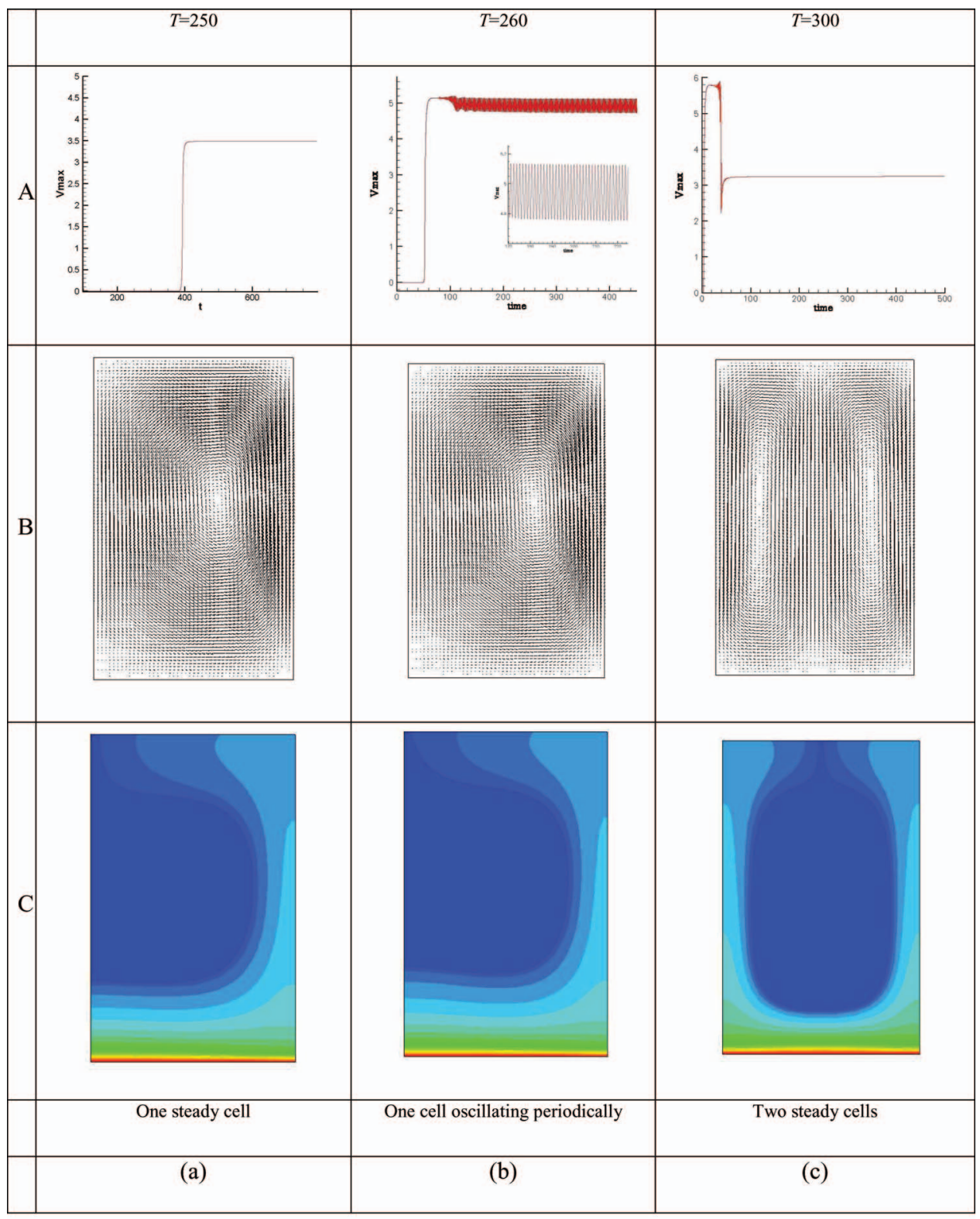

FIG. 15. (Color online) A: Time history of the maximum of velocity in the domain for different values of $T$. B: Velocity field. C: Contour map of the charge density $L=0.614, M=10, C=10$. (a) One steady cell; (b) one cell oscillating periodically; (c) two steady cells.

the appearance of these plumes is related to the destabilization of the laminar sub-layer near the surface. Looking at Figures $18 \mathrm{~B}(\mathrm{~b})$ and $18 \mathrm{C}(\mathrm{b})$, one realizes that there is a thin charged layer close to the injecting electrode where the liquid is almost at rest. If one defines an effective value of $T$ along this layer, it increases with the value of $T$ of the whole layer. When the local value of $T$ overcomes the linear stability criterion $T_{c}$, the laminar sub-layer destabilises, giving place to the emission of charged plumes. A rough estimation gives $1 / 10$ for the sub-layer thickness, giving $T=10 T_{c}$, for the appearance of plumes. 


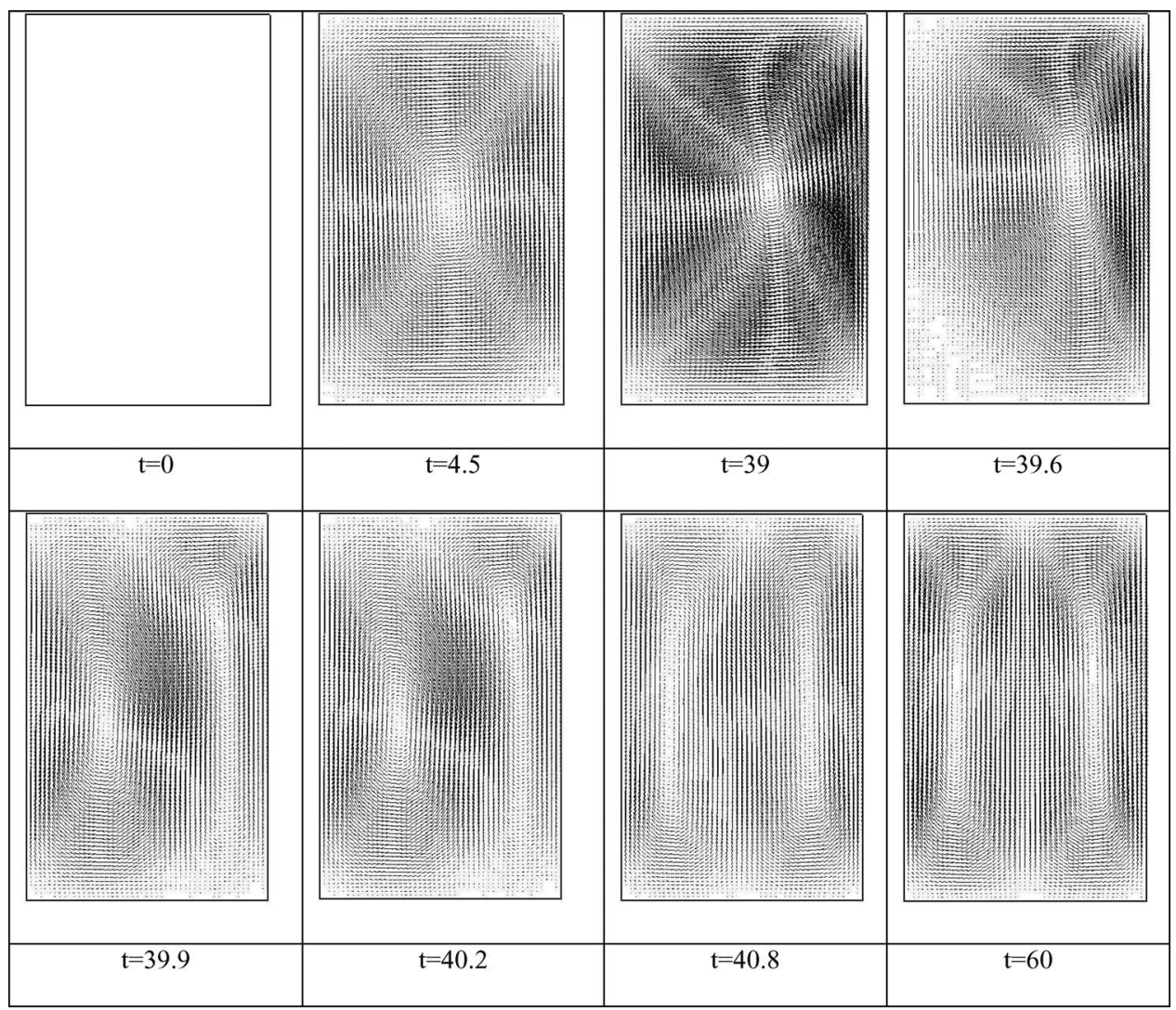

FIG. 16. Velocity field at different time steps. $L=0.614, M=10, C=10, T=300$.

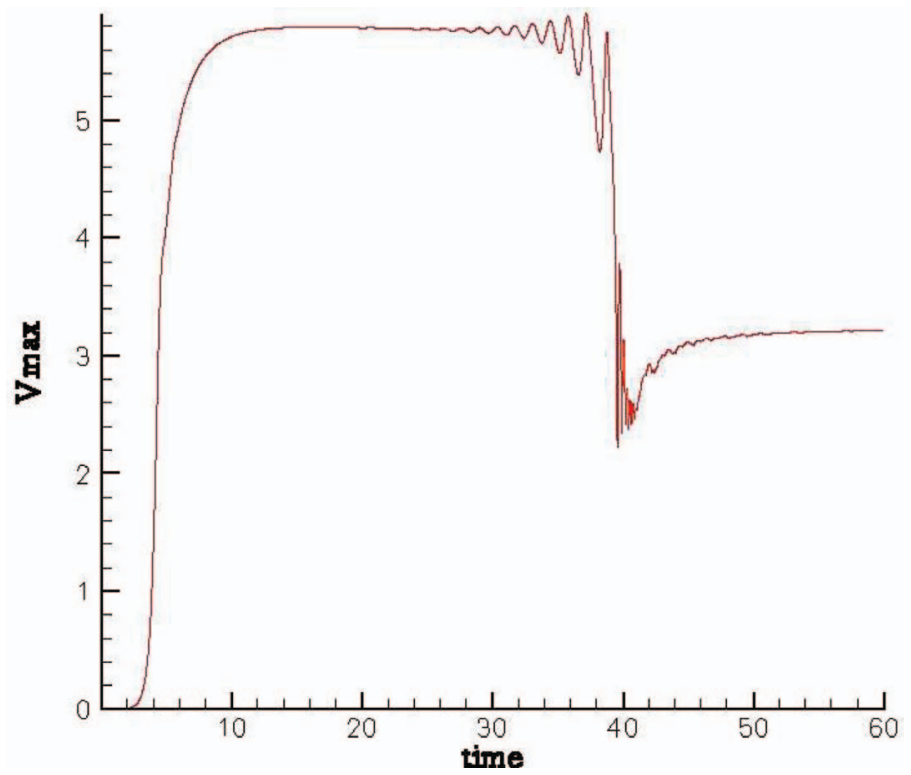

FIG. 17. (Color online) Time history of $V_{\max } . L=0.614, M=10, C=10 T=300$. 


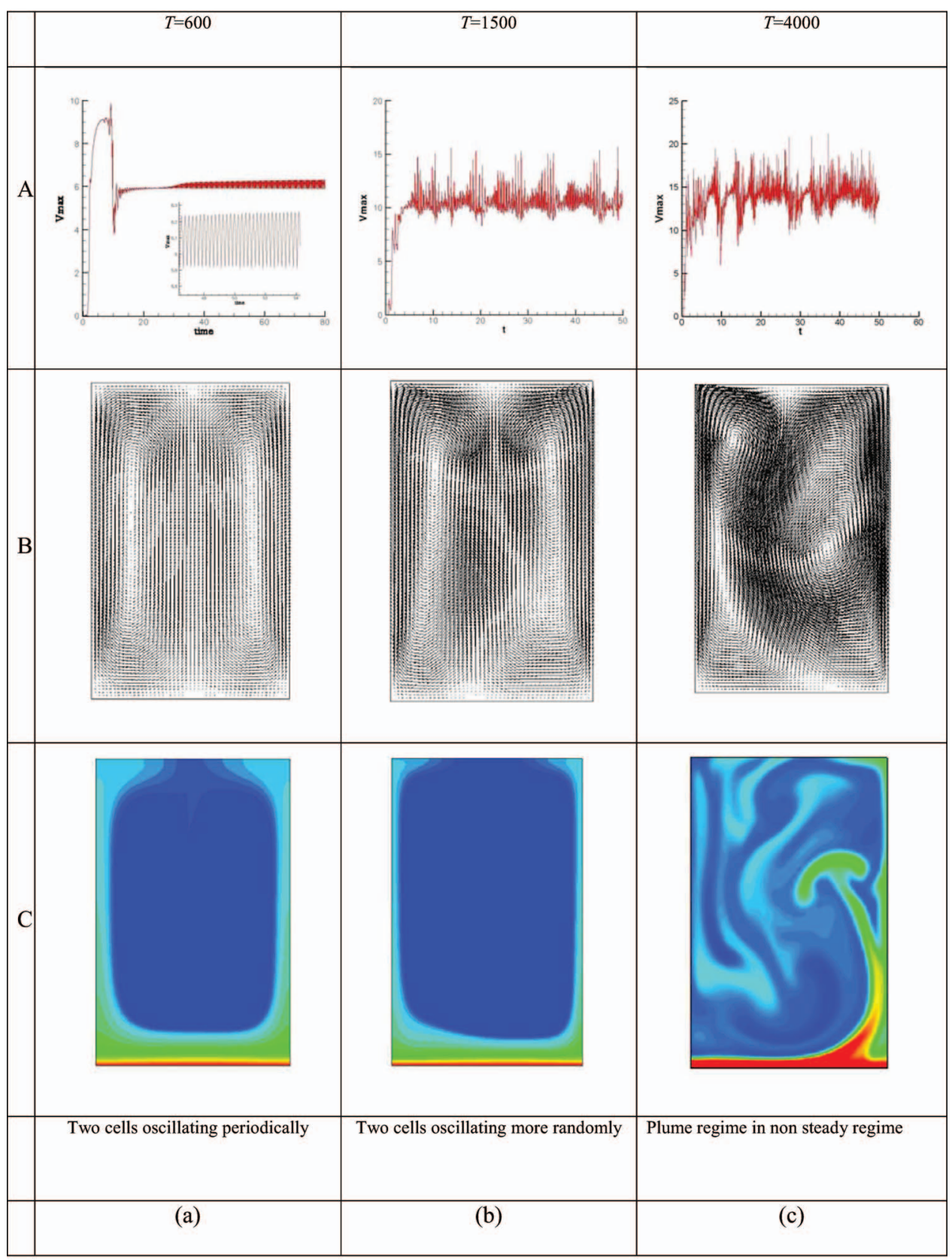

FIG. 18. (Color online) A: Time history of the maximum of velocity in the domain for different values of $T$. B: Velocity field. C: Contour map of the charge density $L=0.614, M=10, C=10$. (a) Two cells oscillating periodically. (b) Two cells oscillating more randomly. (c) Plume regime in non-steady regime.

\section{Electrical Nusselt number}

It is interesting to compute the electrical Nusselt number from the electric current, since experimental data are available in the literature, Lacroix et al. ${ }^{40}$

The electrical Nusselt is defined as: $N_{e}=\frac{I_{e}}{I_{0}}$,

where $I_{e}=\int_{z=\text { constant }} \frac{1}{C} \frac{\partial E_{z}}{\partial t}+q\left(U_{z}+E_{z}\right) d x$ is the current in a non-dimensional form, and $I_{0}$ is the current computed in the case when there is no fluid motion. 


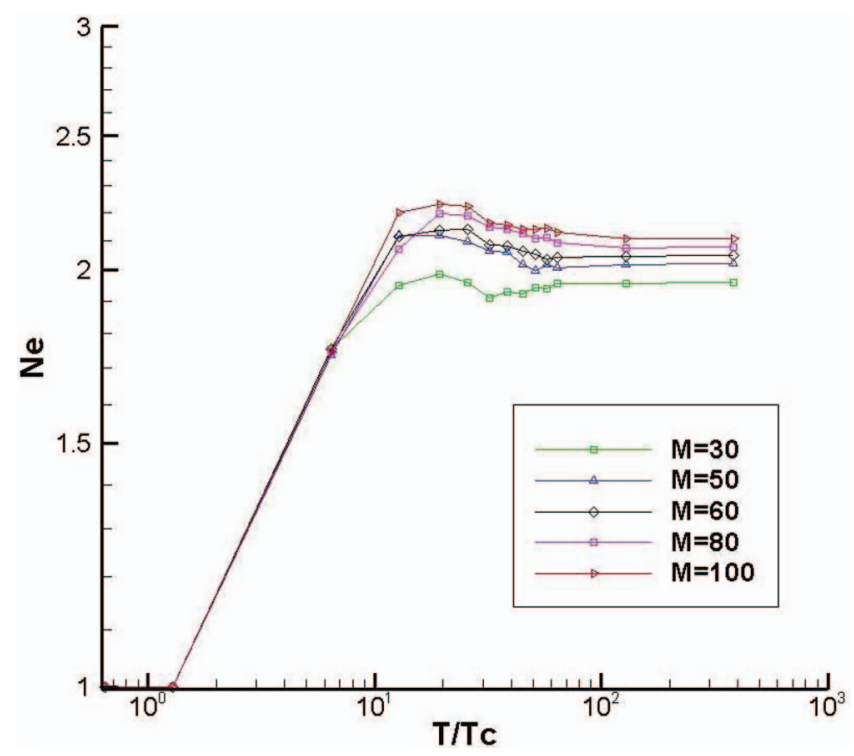

FIG. 19. (Color online) Evolution of the electrical Nusselt number versus $T$.

In our non-dimensional magnitudes, neither $q$ nor $\|\vec{E}\|$ depends explicitly on $T$; therefore, the charge distribution and the electric field computed from a simulation with $T<T_{c}$ correspond to any other value of $T$ in the absence of motion. Since for values of $T$ much greater than the critical value the motion is unsteady, we average $I_{e}$ over long periods of time.

Figure 19 shows the electrical Nusselt number versus $T / T_{c}$ for different values of parameter $M$. This can be compared with the experimental measurements of Lacroix et al. The numerical simulation reproduces some important features of the experimental data. First of all, the Nusselt number saturates for increasing values of $T$. Experimentally, the transition from $\mathrm{Ne}$ increasing with $T$ to saturation corresponds to the transition from viscous to inertial regime. This transition occurs at a value of $T$, which increases with $M$. Although our results are compatible with this behaviour of the transition value, we do not have enough resolution in the computation of $\mathrm{Ne}$ to clearly establish this fact. The origin of this lack of resolution is to be found in the average procedure that we used. Since the motion is very unsteady for high values of $T$, the average provides values that are less and less stable. This can also be seen at the origin of the overshoot observed in the curves Ne versus $T$.

As in the experiments, the value of $T$ at saturation depends on $M$. This fact is analysed in detail by Lacroix et al. ${ }^{40}$ where a scaling law $N e \sim M^{1 / 2}$ is predicted. Although Figure 19 shows that the value of $\mathrm{Ne}$ at saturation increases with $M$, it is difficult to decide whether or not our results follow the scaling law $\mathrm{Ne} \sim M^{1 / 2}$, this uncertainty is probably related to the two-dimensional nature of the simulation. Lacroix et al. ${ }^{40}$ arrived theoretically at this behaviour from two previous relations. One of them is that the velocity scales as $v^{\prime} \sim M E$. This relation is expected to hold in 2D, because it stems from a balance between the inertial and the electric terms in the Navier-Stokes equation. The other relation concerns the ratio between the fluctuations of the charge density and its average value. They obtained $\frac{q^{\prime}}{\bar{q}} \sim M^{-1 / 2}$, where $q^{\prime}$ represents the fluctuations and $\bar{q}$ the temporal average charge density. The production of charge fluctuations is related to the mixing properties of turbulence, and these properties are very different from 2 to 3 dimensions, up to the point at which we cannot properly speak of turbulence in $2 \mathrm{D}$ but rather of a chaotic regime. Therefore, although the saturation of $\mathrm{Ne}$ must be expected in 2D, due to the finite velocity of convection of the electric charge, the dependence on $M$ is not necessarily the same as for 3D. Indeed we must also account the fact that in 2D the flow structure is made of contra-rotating rolls, while in 3D hexagonal cells arise. It would certainly result in a different vortical activity and for this reason a different mechanism of charge mixing which determines the charge transport and, hence, charge distribution which lead consequently to the electrical Nusselt number. This difference in mixing efficiency between 2D 
and 3D fully turbulent expected flow could explain the discrepancy between the level of values of electrical Nusselt number found in our 2D numerical simulations compared to the experimental data of Lacroix et al. ${ }^{40}$ Following the same underlying thought, extending the size domain to $L=10$ (15 cells) instead of $L=0.614$ ( 1 cell) should reduce the discrepancy between the electrical Nusselt number in the numerical experiments compared to experimental data.

\section{E. Spectral analysis}

We have performed a spectral analysis of the fluctuations of the velocity in the unsteady regimes for $M=100$. For a small aspect ratio $(L=0.614)$, the spectra are discrete, as shown in Figure 20, up to values of $T$ of the order 3000. In this range, the spectra show a very sharp central peak and its harmonics. There are also some sub-harmonics. For higher values of $T$, the peaks progressively broaden until the whole spectrum becomes broadband for $T>5000$. Our findings present some differences from the results obtained by Chicón et al. ${ }^{7}$ and Vázquez et al. ${ }^{9}$ Chicón et al. found a broadband spectrum for $L=0.614$. In Ref. 9, the authors compared two different methods, a PIC method similar to the one used by Chicón et al., and the FCT scheme which is more related to our SMART scheme. They found broadband spectra when using the PIC method, but not when using FCT. These differences can be certainly ascribed to the method used to compute the charge density field.

In their experimental study of electroconvection, Malraison et $a .^{41}$ found periodic behaviour but very close to the instability threshold. After this small window of periodicity, there appeared a second frequency and then broadband spectra. The power spectrum decay exponentially with a typical frequency which increases with the applied voltage. Although our simulations show periodic behaviour and broadband behaviour, we have not found the one frequency-two frequencies then the chaos sequence found experimentally. Also the broadband spectra appear for much higher values of $T$ than experimentally. Again, the 2D nature of our simulations may be the reason for these discrepancies.

Figure 21 shows the variation of the frequency of the main peak $f_{0}$ of our spectra as a function of $T$. Apparently there is a change of tendency near $T=3000$. This may be in agreement with the result obtained experimentally by Malraison $e t$ al., where a change in the behaviour in the curve $f_{0}$ versus $V$ was observed at $V=1 \mathrm{kV}$, or some 20 times the critical voltage. Since $T_{c}=150$, the change in behaviour in Figure 21 is in good agreement with the experimental one. This change in slope is related to the change from a viscous to inertial regime, and it is also compatible with the change in behaviour of the electric Nusselt number found in the previous section. The appearance of plumes

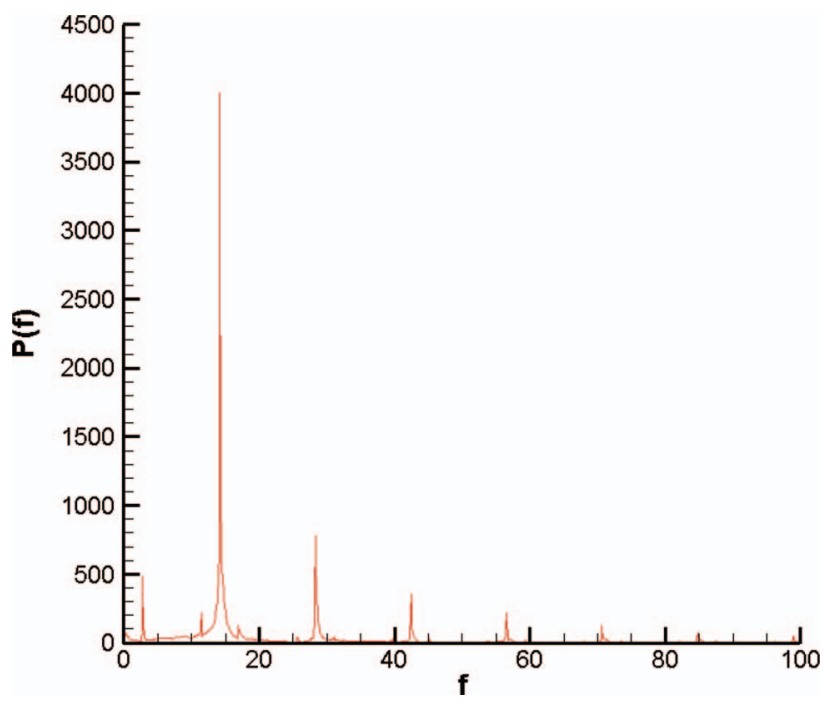

FIG. 20. (Color online) Power spectra for $L=0.614, T=2100, C=10$. 


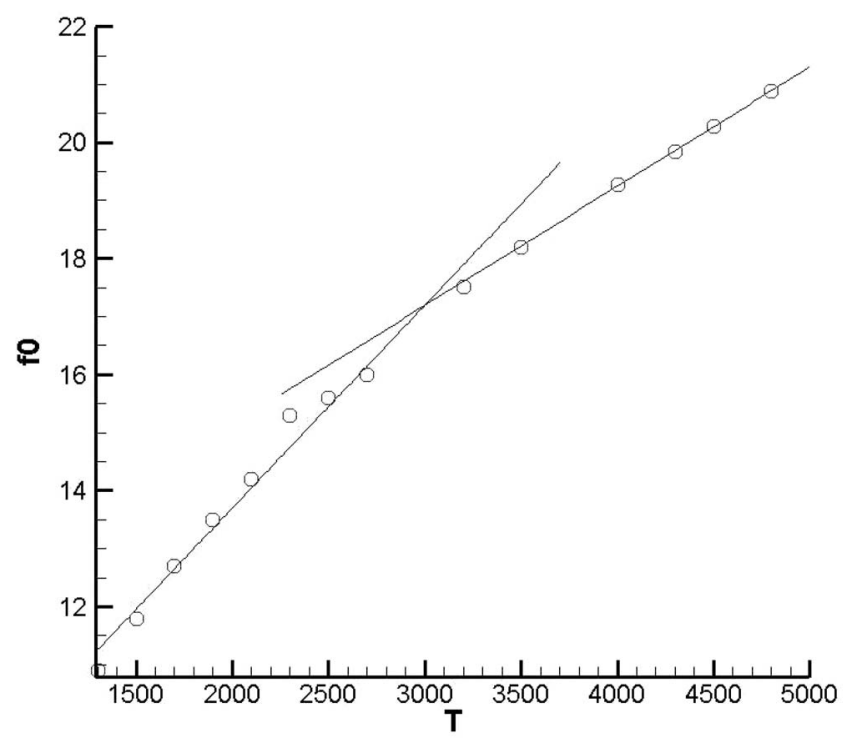

FIG. 21. Characteristic frequency as a function of $T$ for $L=0.614$.

may also be associated with this transition. In any case $f_{0}$ remains proportional to the liquid velocity (Figure 22).

The mechanism of oscillations is related to the chaotic nature of the ion trajectories. These trajectories are shown to be more chaotic when the frequency of oscillation is of the order of the liquid rotation (Pérez et al. ${ }^{42}$ ), which, in turn, is related to the liquid maximum velocity.

For large aspect ratios we always obtain a broadband spectra, and this is in agreement with the experimental findings. The broadband spectra (Figure 23) have an exponential decay of the type $P(f)=P_{0} e^{-f / f_{c}}$. This is also consistent with the experimental results, at least at not very high frequencies. As it is the case for $f_{0}$, the value of $f_{c}$ varies linearly with the mean velocity of the rolls. This is also consistent with the experimental results.

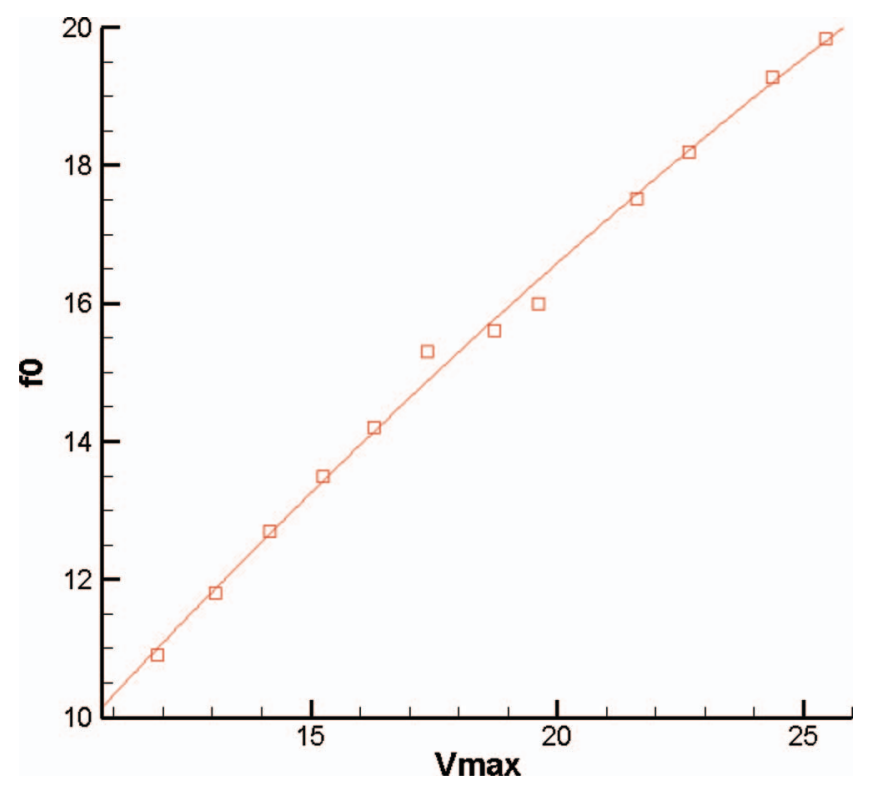

FIG. 22. (Color online) Characteristic frequency as a function of the maximum velocity for $L=0.614$. 


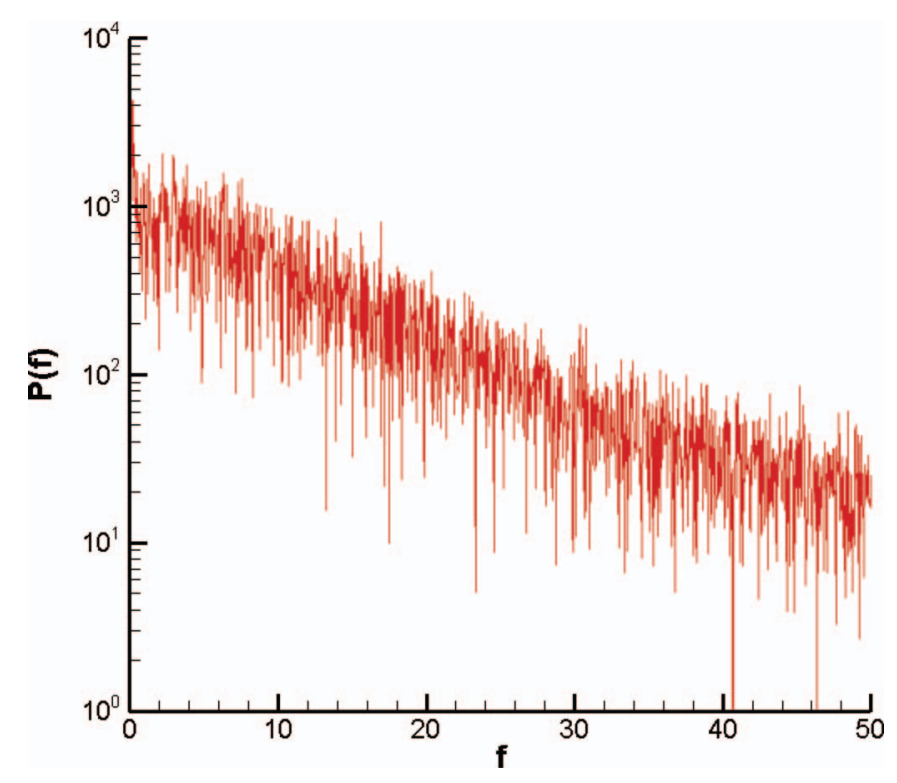

FIG. 23. (Color online) Power spectra for $L=10, T=2100, C=10$.

\section{CONCLUSION}

Numerical simulations have been undertaken to study the electroconvection in a plane layer of a dielectric liquid. The whole set of the coupled hydro and electro dynamic equations have been solved using a second order in time and space accurate finite volume method. The charge density transport equation, which is the critical issue in these problems, has been integrated using a very low diffusing scheme, once the velocity field has been entirely computed from the complete NavierStokes equations including Coulomb force. The capability and the reliability of the numerical code to accurately simulate electroconvection phenomena have been demonstrated through comparison with several stability analysis results. The hysterical behaviour of the maximum velocity versus $T$ could be reproduced, as well as the determination of both the linear and nonlinear criteria in the strong injection case. The scenario for the evolution of the flow structure has been detailed, and different regimes that occur for different characteristic value of the $T$ parameter have been highlighted. It has been found that two stable regimes with one or two cells, according to the value of $T$, occur in strong injection. Further increments of $T$ leads to a regime characterised by the development of charged plumes and, after that, a very unsteady motion.

The evolution of the electrical Nusselt number has been reported and compared with available experimental results. The numerical results are in qualitative accordance with the experimental data. Finally, a spectral analysis of the temporal evolution of the maximum velocity signal has been undertaken. For small aspect ratio domains the spectrum is discrete, whereas for large aspect ratio domains the spectrum is broadband. In all cases, the characteristic frequency of the spectra is proportional to the liquid mean velocity.

\section{ACKNOWLEDGMENTS}

One of the authors (A. T. Pérez) acknowledges financial support from the Spanish Ministerio de Ciencia y Tecnología (MCYT) under Research Project No. FIS2006-03645, and Junta de Andalucía under research projects EXC/2005/FQM-421, P10-FQM-5735, and P09-FQM-4584.

\footnotetext{
${ }^{1}$ N. Felici, "D. C. conduction in liquid dielectrics (part II): Electrohydrodynamic phenomena," Direct Current 2, 147 (1972).

${ }^{2}$ N. Felici, "Phénomènes hydro et aérodynamiques dans la conduction des diélectriques fluides," Revue Gén. Electricité 78, 717 (1969)
} 
${ }^{3}$ P. Atten and J. C. Lacroix, "Non linear hydrodynamic stability of liquids subjected to unipolar injection," J. Mécanique 18, 469 (1979).

${ }^{4}$ A. Castellanos, P. Atten, and A. T. Pérez, "Finite amplitude electroconvection in liquid in the case of weak unipolar injection," PCH, PhysicoChem. Hydrodyn. 9(3/4), 443 (1987).

${ }^{5}$ S. V. Patankar, Numerical Heat Transfer and Fluid Flow (McGraw-Hill, New York, 1980).

${ }^{6}$ A. T. Pérez and A. Castellanos, "Role of charge diffusion in finite-amplitude electroconvection," Phys. Rev. A 40(10), 5844 (1989).

${ }^{7}$ R. Chicón, A. Castellanos, and A. Martin, "Numerical modelling of Coulomb-driven convection in insulating liquids," J. Fluid Mech. 344, 43 (1997).

${ }^{8}$ R. Chicón, A. Castellanos, and A. T. Pérez, "Transient electrohydrodynamic stability of dielectric liquids subjected to unipolar injection,” Inst. Phys. Conf. Ser. 163, 157 (1999).

${ }^{9}$ P. A. Vázquez, G. E. Georghiou, and A. Castellanos, "Characterization of injection instabilities in electrohydrodynamics by numerical modelling: Comparison of particle in cell and flux corrected transport methods for electroconvection between two plates," J. Phys. D 39(13), 2754 (2006).

${ }^{10}$ J. P. Boris and D. L. Book, "Solution of continuity equation by the method of flux corrected transport," Methods Comput. Phys. 16, 85 (1976).

${ }^{11}$ S. T. Zalesak, "Fully multidimensional flux-corrected transport," J. Comput. Phys. 31, 355 (1979).

${ }^{12}$ P. Tsai, Z. A. Daya, V. B. Deyirmenjian, and S. W. Morris, "Direct numerical simulation of supercritical annular electroconvection,” Phys. Rev. E 76, 26305 (2007).

${ }^{13} \mathrm{Ph}$. Traoré, A. T. Pérez, D. Koulova, and H. Romat, "Numerical modelling of finite-amplitude electro-thermo convection in a dielectric liquid layer subjected to both unipolar injection and temperature gradient," J. Fluid Mech. 658, 279 (2010).

${ }^{14}$ R. Glowinski, Numerical Methods for Non Linear Variational Problems (Springer, New York, 1984).

${ }^{15}$ H. Uzawa, "Iterative methods for concave programming," Studies in Linear and Non Linear Programming (Stanford University Press, Stanford, CA, 1958), pp. 154-165.

${ }^{16}$ P. H. Gaskel and A. K. C. Lau, "An assessment of direct stress modelling for elliptic turbulent flows with the aid of a non-diffusive, boundedness-preserving, discretization schemes," in Proceedings of the Fifth International Conference on Numerical Methods in Laminar and Turbulent Flow, Montreal (Pineridge Press, Montreal, Canada, 1987).

${ }^{17}$ P. H. Gaskel and A. K. C. Lau, "Curvature-compensated convective transport: SMART, a new boundedness-preserving transport algorithm,” Int. J. Numer. Methods Fluids 8, 617 (1988).

${ }^{18} \mathrm{Ph}$. Traoré, "Contribution numérique à l'étude des transferts couplés de quantité de mouvement de chaleur et de masse dans un jet semi-confiné," Ph.D. dissertation, University of Toulouse III, France, 1996.

${ }^{19}$ J. R. Melcher, Continuum Electromechanics (MIT, Cambridge, MA, 1981).

${ }^{20}$ A. Castellanos, "Coulomb driven convection in electrohydrodynamics," IEEE Trans. Dielectr. Electr. Insul. 26, 1201 (1991).

${ }^{21}$ P. Atten, "Electrohydrodynamic instability and motion induced by injected space charge in insulating liquids," IEEE Trans. Dielectr. Electr. Insul. 3, 1 (1996).

${ }^{22}$ L. M. Landau and E. M. Lifshitz, Electrodynamics of Continuous Media (Addison-Wesley, RM, Moscow, 1971).

${ }^{23}$ A. Castellanos, "Conduction and polarization," Electrohydrodynamics, edited by A. Castellanos (Springer-Verlag, New York, 1998), Chap. 2.

${ }^{24}$ S. K. Godunov, "A difference scheme for numerical computational of discontinuous solution of hydrodynamic equations," Math Sbornik 47, 271, Translated US Joint Publ. Res. Service. JPRS 7226 (1959).

${ }^{25}$ B. Van Leer, "Towards the ultimate conservative difference scheme. In the quest of monotinicity," Lect. Notes Phys. 18, 163 (1973).

${ }^{26}$ B. Van Leer, "Towards the ultimate conservative difference scheme. II. Monotinicity and conservation combined in a second order scheme," J. Comput. Phys. 14, 361 (1974).

${ }^{27}$ B. Van Leer, “Towards the ultimate conservative difference scheme. V. A second order sequel to Godunov's method," J. Comput. Phys. 32, 101 (1979).

${ }^{28}$ P. D. Lax, Hyperbolic Systems of Conservation Laws and The Mathematical Theory of Shock Waves (SIAM, Philadelphia, 1973).

${ }^{29}$ S. F. Davis, "TVD finite difference schemes and artificial viscosity," ICASE Report 84-20, NASA CR-172373, NASA Langley Research Center (1984).

${ }^{30}$ P. L. Roe, "Generalised formulation of TVD Lax-Wendroff schemes," ICASE Report 84-53, NASA CR-172478, NASA Langley Research Center (1984).

${ }^{31}$ A. Harten, "High resolution schemes for hyperbolic conservation laws," J. Comput. Phys. 49, 357 (1983).

${ }^{32}$ P. K. Sweeby, "High resolution schemes using flux limiters for hyperbolic conservation laws," SIAM J. Numer. Anal. 5, 995 (1984).

${ }^{33}$ F. S. Lien and M. A. Leschziner, "A pressure-velocity solution strategy for compressible flow and its application to shock/boundary-layer interaction using second-moment turbulence closure," J. Fluids Eng. 115(4), 717 (1993).

${ }^{34}$ N. P. Waterson and H. Deconinck, "A unified approach to the design and application of bounded higher-order convection schemes," in Numerical Methods in Laminar and Turbulent Flow: Proceedings of the 9th International Conference, Atlanta, GA, 10-14 July 1995 (Pineridge, Swansea, UK, 1995), vol. 9, Pt. 1 (A97-20701 04-31), pp. 203-214.

${ }^{35}$ P. K. Khosla and S. G. Rubin, "A diagonally dominant second-order accurate implicit scheme," Comput. Fluids 2, 207 (1974).

${ }^{36}$ J. M. Schneider and P. K. Watson, "Electrohydrodynamic stability of space charge limited currents in dielectric liquids. I. Theoretical study," Phys. Fluids 13, 322 (1970).

${ }^{37} \mathrm{P}$. Atten and R. Moreau, "Stabilité électrohydrodynamique des liquides isolants soumis à une injection unipolaire," J. Mécanique 11(3), 471 (1972). 
${ }^{38}$ A. Castellanos and P. Atten, "Numerical modelling of finite amplitude convection of liquids subjected to unipolar injection," IEEE Trans. Ind. Appl. IA-23(5), 825 (1987).

${ }^{39}$ P. A. Vazquez, G. E. Georghiou, and A. Castellanos, "Numerical analysis of the stability of the electrohydrodynamic (EHD) electroconvection between two plates," J. Phys. D 41(17), 175303 (2008).

${ }^{40}$ J. C. Lacroix, P. Atten, and E. J. Hopfinger, "Electro-convection in a dielectric liquid layer subjected to unipolar injection," J. Fluid Mech. 69(3), 539 (1975).

${ }^{41}$ B. Malraison and P. Atten, "Chaotic behavior of instability due to unipolar charge injection in a dielectric liquid," Phys. Rev. Lett. 49, 723 (1982).

${ }^{42}$ A. T. Pérez and A. Castellanos, "Laminar chaotic transport of charge in finite amplitude electroconvection," Phys. Rev. 44, 6659 (1991). 